Pure and Applied Mathematics Quarterly

Volume 2, Number 3

(Special Issue: In honor of

Robert MacPherson, Part 1 of 3)

$699-717,2006$

\title{
Equivariant Giambelli and Determinantal Restriction Formulas for the Grassmannian
}

\author{
V. Lakshmibai ${ }^{\dagger}$, K. N. Raghavan and P. Sankaran \\ Dedicated to Prof. Robert MacPherson
}

\begin{abstract}
The main result of the paper is a determinantal formula for the restriction to a torus fixed point of the equivariant class of a Schubert subvariety in the torus equivariant integral cohomology ring of the Grassmannian. As a corollary, we obtain an equivariant version of the Giambelli formula.
\end{abstract}

The (torus) equivariant cohomology rings of flag varieties in general and of the Grassmannian in particular have recently attracted much interest. Here we consider the equivariant integral cohomology ring of the Grassmannian. Just as the ordinary Schubert classes form a module basis over the ordinary cohomology ring of a point (namely the ring of integers) for the ordinary integral cohomology ring of the Grassmannian, so do the equivariant Schubert classes form a basis over the equivariant cohomology of a point (namely the ordinary cohomology ring of the classifying space of the torus) for the equivariant cohomology ring (this is true for any generalized flag variety of any type, not just the Grassmannian). Again as in the ordinary case, computing the structure constants of the multiplication with respect to this basis is an interesting problem that goes by the name of Schubert calculus. There is a forgetful functor from equivariant cohomology to ordinary cohomology so that results about the former specialize to those about the latter.

Knutson-Tao-Woodward [5] and Knutson-Tao [6] show that the structure constants, both ordinary and equivariant, count solutions to certain jigsaw puzzles, thereby showing that they are "manifestly" positive. In the present paper we take a very different route to computing the equivariant structure constants. Namely,

Received June 9, 2005.

2000 Mathematics Subject Classification14M15

$\dagger$ Partially supported by NSF grant DMS 0400679 and NSA-MDA 904-03-1-0034. 
we try to extend to the equivariant case the classical approach by means of the Pieri and Giambelli formulas. Recall, from [3, Eq.(10), p.146] for example, that the Giambelli formula expresses an arbitrary Schubert class as a polynomial with integral coefficients in certain "special" Schubert classes - the Chern classes of the tautological quotient bundle - and that the Pieri formula expresses as a linear combination of the Schubert classes the product of a special Schubert class with an arbitrary Schubert class. Together they can be used to compute the structure constants.

We only partially succeed in our attempt: the first of the three theorems of this paper - see $\S 2$ below - is an equivariant Giambelli formula that specializes to the ordinary Giambelli formula as in [3, Eq.(10), p.146], but we still do not have a satisfactory equivariant Pieri formula - see, however, $\S 7$ below. The derivation in Fulton $[2, \S 14.3]$ of the Giambelli formula can perhaps be extended to the equivariant case, but this is not what we do. Instead, we deduce the Giambelli formula from our second theorem which gives a certain closed-form determinantal formula for the restriction to a torus fixed point of an equivariant Schubert class.

This "restriction formula" (Theorem 2 in $\S 3$ below) is the point of this papermore so than the Giambelli, for among other things it might also hold the key to the Pieri. It in turn is deduced from Theorem 3 which can be paraphrased thus: a Gröbner degeneration of an open piece around a torus fixed point of a torus stable subvariety computes the restriction to the fixed point of the equivariant cohomology class of the subvariety. Recall that a Gröbner degeneration comes from a Gröbner basis. It is a 1-parameter flat degeneration. See $\S 6$ for the precise meaning. Such Gröbner degenerations at torus fixed points of Schubert subvarieties in the Grassmannian have recently been obtained [9, 7, 8, 10]. Combining this result about degenerations with Theorem 3 yields a proof of Theorem 2 .

As pointed out to us by the referee, Theorem 3 is well known. The precise references (as indicated by the referee) are given in $\S 6$. The passage from Theorem 3 plus the result about degenerations to the restriction formula involves only an elementary combinatorial inductive argument. (Is there an elegant LindstromGessel-Viennot type argument for this passage? We do not know.) The passage from the restriction formula to the Giambelli again involves only elementary matrix manipulations.

Acknowledgments: Parts of this work were done during visits of the first named author to Chennai Mathematical Institute and of the other two authors to The Abdus Salam International Centre for Theoretical Physics. The hospitality of the two institutions is gratefully acknowledged. It is with great pleasure that we thank the referee for a quick and thorough reading of the manuscript and for the insightful comments; thanks in particular for indicating how Theorem 3 follows from results available in the literature. 


\section{THE SET UP}

Fix once and for all two positive integers $d$ and $n$ with $d \leq n$. Let $V$ be an $n$ dimensional complex vector space, and $\mathbb{G}_{d, n}$ the Grassmannian of $d$-dimensional linear subspaces of $V$. The defining action of the general linear group $G L(V)$ on $V$ induces an action on $\mathbb{G}_{d, n}$. We are interested in the $T$-equivariant integral cohomology ring $\mathcal{H}$ of $\mathbb{G}_{d, n}$, where $T$ is a fixed maximal torus of $G L(V)$.

We refer to $[6, \S 2]$ and the references in that paper for the details that we leave out in this section.

The natural map from $\mathbb{G}_{d, n}$ to $\operatorname{Spec}(\mathbb{C})$ induces an $\mathcal{S}$-algebra structure on $\mathcal{H}$, where $\mathcal{S}:=H_{T}^{*}(\operatorname{Spec}(\mathbb{C}))$ is the $T$-equivariant integral cohomology ring of $\operatorname{Spec}(\mathbb{C})$ (namely the ordinary integral cohomology ring of the classifying space of $T$ ). The $\mathcal{S}$-algebra $\mathcal{H}$ is independent of the choice of $T$ because any two maximal tori in $G L(V)$ are conjugate.

The choice of a maximal torus $T$ amounts to the choice of an unordered vector space basis $\mathcal{B}$ of $V$ : the elements of $T$ are precisely those invertible linear transformations for which each element of $\mathcal{B}$ is an eigenvector. Each element $b$ of $\mathcal{B}$ thus defines a character $\epsilon_{b}$ of $T$ that sends elements of $T$ to their respective eigenvalues with respect to $b$. The collection $\left\{\epsilon_{b} \mid b \in \mathcal{B}\right\}$ forms an integral basis for the group $X(T)$ of characters of $T$. The ring $\mathcal{S}$ is graded isomorphic to the symmetric algebra of the abelian group $X(T)$ with $X(T)$ living in degree 2 . We may therefore identify $\mathcal{S}$ with the polynomial ring $\mathbb{Z}\left[\epsilon_{b} \mid b \in \mathcal{B}\right]$, where the $\epsilon_{b}$ are variables in degree 2 .

Since $\mathbb{G}_{d, n}$ is a smooth variety on which $T$ acts algebraically with finitely many fixed points, it follows that $\mathcal{H}$ is a free $\mathcal{S}$-module with basis the (equivariant) classes of the Schubert subvarieties. These subvarieties are defined with respect to a fixed Borel subgroup $B$ containing $T$ : they are the closures of the $B$-orbits in $\mathbb{G}_{d, n}$. The formulas of this paper are independent of the choice of $B$ because any two such Borel subgroups are conjugate by an element in the normalizer of $T$ in $G L(V)$.

The choice of a Borel subgroup $B$ containing $T$ amounts to the choice of an ordering on the elements of the basis $\mathcal{B}$. Let $b_{1}, \ldots, b_{n}$ be the elements of $\mathcal{B}$ thus ordered. We have $\mathcal{S}=\mathbb{Z}\left[\epsilon_{1}, \ldots, \epsilon_{n}\right]$, where $\epsilon_{j}:=\epsilon_{b_{j}}$.

There is a one-to-one correspondence between the $B$-orbits and the $T$-fixed points in $\mathbb{G}_{d, n}$ : each $B$-orbit contains one and only one $T$-fixed point. The $T$ fixed points are indexed by the subsets of cardinality $d$ of $\mathcal{B}$.

Denote by $I(d, n)$ the set of subsets of cardinality $d$ of $\{1, \ldots, n\}$. We use $u, v, w, \ldots$ to denote elements of $I(d, n)$. For $u$ in $I(d, n)$, we write $u=\left(u_{1}, \ldots, u_{d}\right)$ 
where $u_{1}, \ldots, u_{d}$ are the elements of $u$ arranged in increasing order: $1 \leq u_{1}<$ $\ldots<u_{d} \leq n$.

Given $u=\left(u_{1}, \ldots, u_{d}\right)$ in $I(d, n)$, denote by $e^{u}$ the $T$-fixed point of $\mathbb{G}_{d, n}$ that is the span of $\left\{b_{u_{1}}, \ldots, b_{u_{d}}\right\}$, by $X(u)$ the closure of the $B$-orbit containing $e^{u}$, by $[X(u)]$ the $T$-equivariant class in $\mathcal{H}$ of the Schubert subvariety $X(u)$, and by $[X(u)]_{\mathrm{cl}}$ the ordinary cohomology class of $X(u)$.

For each $T$-fixed point $e^{v}, v$ in $I(d, n)$, we have a natural "restriction" map $\operatorname{Res}_{v}: \mathcal{H}:=H_{T}^{*}\left(\mathbb{G}_{d, n}\right) \rightarrow \mathcal{S}:=H_{T}^{*}\left(e^{v}\right)$ induced by the inclusion of $\left\{e^{v}\right\}$ in $\mathbb{G}_{d, n}$. The direct product of these is an injection of rings:

$$
\prod \operatorname{Res}_{v}: \mathcal{H} \hookrightarrow \prod_{v \in I(d, n)} H_{T}^{*}\left(e^{v}\right)
$$

For $u$ and $v$ in $I(d, n)$, denote by $\left.[X(u)]\right|_{v}$ the image in $\mathcal{S}$ under $\operatorname{Res}_{v}$ of the equivariant class $[X(u)]$. The image of $\mathcal{H}$ under $\prod \operatorname{Res}_{v}$ has a neat description but we will have no use for this here: a tuple $\left(\alpha_{v}\right)_{v \in I(d, n)}$ in $\prod_{I(d, n)} \mathcal{S}$ belongs to the image of $\mathcal{H}$ under $\prod \operatorname{Res}_{v}$ if and only if, whenever $w$ and $x$ in $I(d, n)$ are so related that there exist integers $i$ and $j, 1 \leq i, j \leq n$, with $x=(w \cup\{j\}) \backslash\{i\}$, it holds that $\epsilon_{j}-\epsilon_{i}$ divides $\alpha_{x}-\alpha_{w}$.

\section{An equivariant Giambelli formula}

Given $u=\left(u_{1}, \ldots, u_{d}\right)$ in $I(d, n)$, set

$$
\lambda_{1}:=n-d+1-u_{1}, \quad \ldots \quad \lambda_{i}:=n-d+i-u_{i}, \quad \ldots \quad \lambda_{d}:=n-u_{d} .
$$

Then $n-d \geq \lambda_{1} \geq \lambda_{2} \geq \ldots \geq \lambda_{d} \geq 0$.

If $u$ is such that $\lambda_{2}=\ldots=\lambda_{d}=0$, we call the Schubert variety $X(u)$ and its cohomology class special; furthermore the equivariant and ordinary cohomology classes $[X(u)]$ and $[X(u)]_{\mathrm{cl}}$ are denoted instead by $\left[\lambda_{1}\right]$ and $\left[\lambda_{1}\right]_{\mathrm{cl}}$. We extend the terminology and notation to all integers by setting $[p]:=0$ if $p$ is outside the range $0,1, \ldots, n-d$.

Observe that $[p]$ belongs to $H_{T}^{2 p}\left(\mathbb{G}_{d, n}\right)$, which explains the notation.

The classical Giambelli formula gives an expression for an arbitrary Schubert class in the ordinary cohomology ring of the Grassmannian $\mathbb{G}_{d, n}$ as the determinant of a $d \times d$ matrix whose entries are special classes. For $u=\left(u_{1}, \ldots, u_{d}\right)$ in 
$I(d, n)$, we have, from [3, Eq.(10), page146] for example, $[X(u)]_{\mathrm{cl}}=$

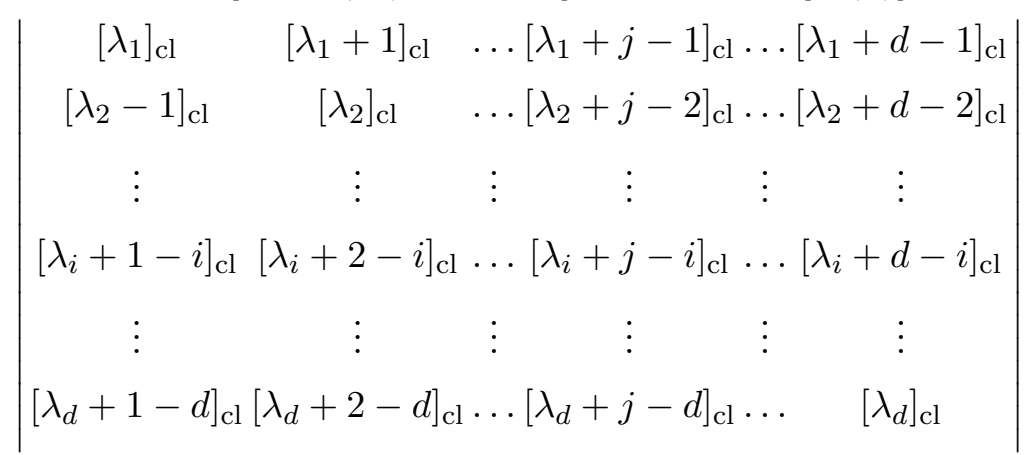

The $i$ th entry on the main diagonal is $\left[\lambda_{i}\right]$ and the index increases by 1 per column as we move rightwards in the same row. The subscript "cl" is to remind us that the classes are in ordinary cohomology. The theorem below gives an equivariant version of the above formula.

The proof of the equivariant version does not use the ordinary version of the formula. In fact, it gives another proof of the ordinary version by specialization.

Let $u=\left(u_{1}, \ldots, u_{d}\right)$ in $I(d, n)$. For $i, j$ integers such that $1 \leq i, j \leq d$, set

$$
u[i, j]=\sum_{k=0}^{j-1} c\left(u_{i}, j, k\right)\left[\lambda_{i}+j-i-k\right]
$$

where $c\left(u_{i}, j, k\right):=(-1)^{k} h_{k}\left(\epsilon_{u_{i}-j+1+k}, \ldots, \epsilon_{u_{i}}\right)$-here $h_{k}$ is the "complete symmetric polynomial," the sum of all monomials of degree $k$ in the elements $\epsilon_{u_{i}-j+1+k}$, $\ldots, \epsilon_{u_{i}}$ of $H_{T}^{*}(\mathrm{pt})=\mathcal{S}$. If $u_{i}-j+1+k \leq 0$, then $c\left(u_{i}, j, k\right)$ does not make sense, but this does not matter since $\lambda_{i}+j-i-k \geq n-d+1$ and so $\left[\lambda_{i}+j-i-k\right]=0$ by definition.

Theorem 1. With notation as above, given $u=\left(u_{1}, \ldots, u_{d}\right)$ in $I(d, n)$, the equivariant cohomology class $[X(u)]$ is the determinant of the $d \times d$ matrix whose $(i, j)$ th entry is $u[i, j]$.

This theorem will be deduced in $\S 4$ from the restriction formula (Theorem 2) and the injection (1).

\section{A DETERMinANTAL FORMUla FOR THE RESTRICTION}

For integers $p, k, r$, set

$$
\mu_{r}^{k}(p):=\prod_{j=k, k+1, \ldots, r} \epsilon(j, p)
$$

where $\epsilon(j, p):=\epsilon_{j}-\epsilon_{p}$. This is well-defined as an element of the polynomial ring $\mathcal{S}$ only when $p, k$, and $r$ belong to the range $1,2, \ldots, n$ and $k \leq r$, but it is 
convenient to extend the notation somewhat: if $k=n+1$, the product, being over an empty index set, is taken to be 1 .

Theorem 2. Given $u=\left(u_{1}, \ldots, u_{d}\right)$ and $v=\left(v_{1}, \ldots, v_{d}\right)$ belonging to $I(d, n)$, the restriction $\left.[X(u)]\right|_{v}$ of the T-equivariant cohomology class $[X(u)]$ of the Schubert variety $X(u)$ in the Grassmannian $\mathbb{G}_{d, n}$ to the $T$-fixed point $e^{v}$ determined by $v$ equals

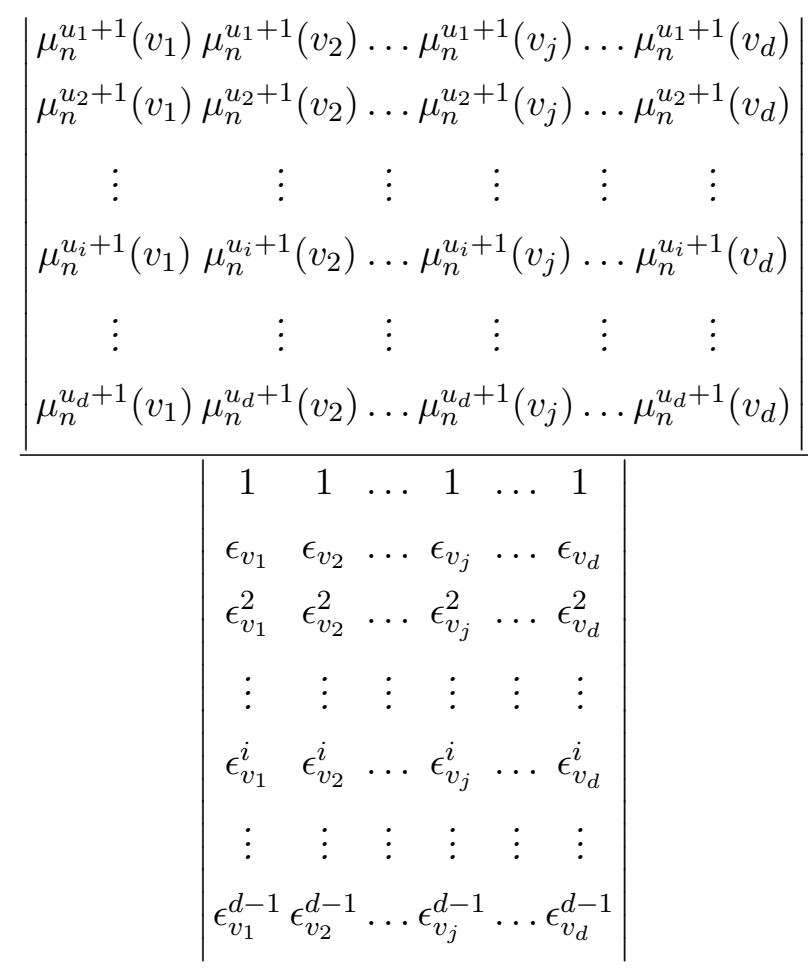

The denominator in the above expression for $\left.[X(u)]\right|_{v}$ is the Vandermonde determinant which equals

$$
\epsilon\left(v_{2}, v_{1}\right) \cdot\left(\epsilon\left(v_{3}, v_{1}\right) \epsilon\left(v_{3}, v_{2}\right)\right) \cdot \ldots \cdot\left(\epsilon\left(v_{d}, v_{1}\right) \ldots \epsilon\left(v_{d}, v_{d-1}\right)\right)
$$

The proof of this theorem occupies sections 5 and 6 .

\section{Proof of the equivariant Giambelli}

In this section, Theorem 1 is deduced from Theorem 2. Because of the injection $(1)$, it is enough to show that, for an arbitrary $v=\left(v_{1}, \ldots, v_{d}\right)$ in $I(d, n)$, the restriction $\left.[X(u)]\right|_{v}$ is the determinant of the $d \times d$ matrix whose $(i, j)$ th entry is $\left.u[i, j]\right|_{v}$. We first obtain a determinantal formula for $\left.u[i, j]\right|_{v}$ :

$$
\left.u[i, j]\right|_{v}=\operatorname{det}(N) / \mathfrak{V}(v),
$$


where

$$
\mathfrak{V}(v):=\left(\epsilon_{v_{2}}-\epsilon_{v_{1}}\right) \cdot\left(\epsilon_{v_{3}}-\epsilon_{v_{1}}\right)\left(\epsilon_{v_{3}}-\epsilon_{v_{2}}\right) \cdot \ldots \cdot\left(\epsilon_{v_{d}}-\epsilon_{v_{1}}\right) \cdots\left(\epsilon_{v_{d}}-\epsilon_{v_{d-1}}\right)
$$

( $\mathfrak{V}$ stands for Vandermonde) and $N$ denotes the following matrix (see $\S 2$ for definition of $\left.\mu_{r}^{k}(p)\right)$

$$
\left(\begin{array}{ccccc}
\mu_{n}^{u_{i}+1}\left(v_{1}\right)\left(-\epsilon_{v_{1}}\right)^{j-1} & \ldots \mu_{n}^{u_{i}+1}\left(v_{s}\right)\left(-\epsilon_{v_{s}}\right)^{j-1} & \ldots \mu_{n}^{u_{i}+1}\left(v_{d}\right)\left(-\epsilon_{v_{d}}\right)^{j-1} \\
\mu_{n}^{n-d+3}\left(v_{1}\right) & \ldots & \mu_{n}^{n-d+3}\left(v_{s}\right) & \ldots & \mu_{n}^{n-d+3}\left(v_{d}\right) \\
\vdots & \vdots & \vdots & \vdots & \vdots \\
\mu_{n}^{n-d+r+1}\left(v_{1}\right) & \ldots & \mu_{n}^{n-d+r+1}\left(v_{s}\right) & \ldots & \mu_{n}^{n-d+r+1}\left(v_{d}\right) \\
\vdots & \vdots & \vdots & \vdots & \vdots \\
\mu_{n}^{n}\left(v_{1}\right) & \ldots & \mu_{n}^{n}\left(v_{s}\right) & \ldots & \mu_{n}^{n}\left(v_{d}\right) \\
1 & \ldots & 1 & \ldots & 1
\end{array}\right)
$$

To prove (4), we substitute for the restrictions of the special classes on the right side of (2) the determinantal expressions given by Theorem 2 :

$$
\begin{aligned}
\left.u[i, j]\right|_{v} & =\left.\sum_{k=0}^{j-1} c\left(u_{i}, j, k\right)\left[\lambda_{i}+j-i-k\right]\right|_{v} \\
& =\sum_{k=0}^{j-1} c\left(u_{i}, j, k\right) \frac{\operatorname{det}\left(\mathfrak{N u m}\left(\lambda_{i}+j-i-k\right)\right)}{\mathfrak{V}(v)}
\end{aligned}
$$

where we have written $\mathfrak{N u m}\left(\lambda_{i}+j-i-k\right)$ for the $d \times d$ matrix whose determinant is the numerator of the expression for $\left.\left[\lambda_{i}+j-i-k\right]\right|_{v}$ given by Theorem 2 . Rows 2 through $d$ of $\mathfrak{N u m}\left(\lambda_{i}+j-i-k\right)$ do not change as $k$ varies: they are the same as the corresponding ones of the matrix $N$ in (4). And the first row of $\mathfrak{N u m}\left(\lambda_{i}+j-i-k\right)$ is

$$
\left(\mu_{n}^{u_{i}-j+k+2}\left(v_{1}\right), \ldots, \mu_{n}^{u_{i}-j+k+2}\left(v_{d}\right)\right) .
$$

So (4) follows once we prove

$$
\sum_{k=0}^{j-1} c\left(u_{i}, j, k\right) \mu_{n}^{u_{i}-j+k+2}\left(v_{s}\right)=\left(-\epsilon_{v_{s}}\right)^{j-1} \mu_{n}^{u_{i}+1}\left(v_{s}\right) .
$$

The above identity is the special case $m=j-1$ of the following more general identity: for $0 \leq m \leq j-1$,

$$
\begin{aligned}
& \sum_{k=0}^{m}(-1)^{k} h_{k}\left(\epsilon_{u_{i}-j+1+k}, \ldots, \epsilon_{u_{i}}\right) \mu_{n}^{u_{i}-j+k+2}\left(v_{s}\right)= \\
& (-1)^{m} h_{m}\left(\epsilon_{v_{s}}, \epsilon_{u_{i}-j+2+m}, \ldots, \epsilon_{u_{i}}\right) \mu_{n}^{u_{i}-j+m+2}\left(v_{s}\right)
\end{aligned}
$$


The proof of (6) is by induction on $m$. First note that it holds for $m=0$. Now for the induction step: assuming that it is true for $m$, we show it holds for $m+1$. Taking $(-1)^{m+1} \mu_{n}^{u_{i}-j+m+3}\left(v_{s}\right)$ common out of the two terms in the following

$$
\begin{aligned}
(-1)^{m} & h_{m}\left(\epsilon_{v_{s}}, \epsilon_{u_{i}-j+2+m}, \ldots, \epsilon_{u_{i}}\right) \mu_{n}^{u_{i}-j+m+2}\left(v_{s}\right)+ \\
& (-1)^{m+1} h_{m+1}\left(\epsilon_{u_{i}-j+2+m}, \ldots, \epsilon_{u_{i}}\right) \mu_{n}^{u_{i}-j+m+3}\left(v_{s}\right)
\end{aligned}
$$

we need only show that

$$
\begin{array}{r}
-h_{m}\left(\epsilon_{v_{s}}, \epsilon_{u_{i}-j+2+m}, \ldots, \epsilon_{u_{i}}\right) \cdot\left(\epsilon_{u_{i}-j+m+2}-\epsilon_{v_{s}}\right) \\
+h_{m+1}\left(\epsilon_{u_{i}-j+2+m}, \ldots, \epsilon_{u_{i}}\right) \\
=h_{m+1}\left(\epsilon_{v_{s}}, \epsilon_{u_{i}-j+3+m}, \ldots, \epsilon_{u_{i}}\right)
\end{array}
$$

but this is just the sum of the following two elementary equalities:

$$
\begin{aligned}
h_{m+1}\left(\epsilon_{v_{s}}, \epsilon_{u_{i}-j+3+m}, \ldots, \epsilon_{u_{i}}\right)= & h_{m+1}\left(\epsilon_{v_{s}}, \epsilon_{u_{i}-j+2+m}, \ldots, \epsilon_{u_{i}}\right) \\
& -\epsilon_{u_{i}-j+2+m} h_{m}\left(\epsilon_{v_{s}}, \epsilon_{u_{i}-j+2+m}, \ldots, \epsilon_{u_{i}}\right) \\
h_{m+1}\left(\epsilon_{v_{s}}, \epsilon_{u_{i}-j+2+m}, \ldots, \epsilon_{u_{i}}\right)= & h_{m+1}\left(\epsilon_{u_{i}-j+2+m}, \ldots, \epsilon_{u_{i}}\right) \\
& +\epsilon_{v_{s}} h_{m}\left(\epsilon_{v_{s}}, \epsilon_{u_{i}-j+2+m}, \ldots, \epsilon_{u_{i}}\right) .
\end{aligned}
$$

Now that (4) is proved, we proceed with the proof of the theorem. If we delete the 1st row and sth column of $N$, the determinant of the resulting sub-matrix is

$$
\mathfrak{V}\left(v_{1}, \ldots, \hat{v}_{s}, \ldots, v_{d}\right):=\frac{\mathfrak{V}(v)}{\epsilon\left(v_{s}, v_{1}\right) \ldots \epsilon\left(v_{s}, v_{s-1}\right) \cdot \epsilon\left(v_{s+1}, v_{s}\right) \ldots \epsilon\left(v_{d}, v_{s}\right)} .
$$

This follows since the determinant has degree $0+1+\ldots+d-2$ in the epsilons and is divisible by $\epsilon\left(v_{j}, v_{i}\right)$ for $1 \leq i, j \leq n, i, j \neq s$. For the determinant of $N$, expanding by the first row, we thus obtain

$$
\operatorname{det}(N)=\sum_{s=1}^{d} \mu_{n}^{u_{i}+1}\left(v_{s}\right) \epsilon_{v_{s}}^{j-1} \mathfrak{V}\left(v_{1}, \ldots, \hat{v}_{s}, \ldots, v_{d}\right)
$$

Observe that the right side is the product of the row matrix

$$
\left(\mu_{n}^{u_{i}+1}\left(v_{1}\right), \ldots, \mu_{n}^{u_{i}+1}\left(v_{d}\right)\right)
$$

with the column matrix whose transpose is

$$
\left(\epsilon_{v_{1}}^{j-1} \mathfrak{V}\left(\hat{v}_{1}, v_{2}, \ldots, v_{d}\right), \ldots, \epsilon_{v_{s}}^{j-1} \mathfrak{V}\left(v_{1}, \ldots, v_{d-1}, \hat{v}_{d}\right)\right)
$$


This means the following for the matrix-let us call it $M$ - whose $(i, j)$ th entry is $\left.u[i, j]\right|_{v}: \mathfrak{V}(v) M$ equals

$$
\left(\begin{array}{ccc}
\mu_{n}^{u_{1}+1}\left(v_{1}\right) \ldots \mu_{n}^{u_{1}+1}\left(v_{d}\right) \\
\vdots & \vdots & \vdots \\
\mu_{n}^{u_{d}+1}\left(v_{1}\right) \ldots \mu_{n}^{u_{d}+1}\left(v_{d}\right)
\end{array}\right)\left(\begin{array}{ccc}
\epsilon_{v_{1}}^{0} \mathfrak{V}\left(\hat{v}_{1}, \ldots, v_{d}\right) \ldots \epsilon_{v_{1}}^{d-1} \mathfrak{V}\left(\hat{v}_{1}, \ldots, v_{d}\right) \\
\vdots & \vdots & \vdots \\
\epsilon_{v_{s}}^{0} \mathfrak{V}\left(v_{1}, \ldots, \hat{v}_{d}\right) \ldots \epsilon_{v_{s}}^{d-1} \mathfrak{V}\left(v_{1}, \ldots, \hat{v}_{d}\right)
\end{array}\right)
$$

Since $\prod_{s=1}^{d} \mathfrak{V}\left(v_{1}, \ldots, \hat{v}_{s}, \ldots, v_{d}\right)=\mathfrak{V}(v)^{d-2}$, the determinant of the matrix on the right above is $\mathfrak{V}(v)^{d-1}$. The matrix on the left - let us call it $P$-is the numerator in the formula for $\left.[X(u)]\right|_{v}$ of Theorem 2. Taking determinants, we get

$$
\mathfrak{V}(v)^{d} \operatorname{det}(M)=\operatorname{det}(P) \mathfrak{V}(v)^{d-1}
$$

and so by Theorem 2

$$
\operatorname{det}(M)=\operatorname{det}(P) / \mathfrak{V}(v)=\left.[X(u)]\right|_{v}
$$

\section{Proof of the RESTRICTION FORMUlA}

In this section, Theorem 2 is proved. Theorem 3, which is stated and proved in $\S 6$, allows us to reduce the proof to combinatorics. More precisely, Theorem 3 tells us that if we have a Gröbner degeneration of an open piece of the Schubert variety $X(u)$ around the $T$-fixed point $e^{v}$, then we can compute the desired restriction $\left.[X(u)]\right|_{v}$. Such a Gröbner degeneration is described in [7]-indeed it was the goal of that paper to describe such a degeneration. We now recall this description.

We identify $\mathbb{G}_{d, n}$ as the orbit space for the action on $n \times d$ matrices of rank $d$ by the group of invertible $d \times d$ matrices by multiplication on the right. The subset consisting of those matrices in which the submatrix determined by the rows $v_{1}, \ldots, v_{d}$ is the identity matrix gives us an affine $T$-stable patch of $\mathbb{G}_{d, n}$ around the point $e^{v}$. This patch is an affine space which we denote $\mathbb{A}^{v}$. The coordinate function $X(r, j)$ on $\mathbb{A}^{v}$ determined by the entry of the matrix in position $(r, j)$, $r \notin v$, is an eigenvector for $T$ with character $-\left(\epsilon_{r}-\epsilon_{v_{j}}\right)$. Thus a natural way to index these coordinates on $\mathbb{A}^{v}$ is by the pairs $(r, c), 1 \leq r, c \leq n$, such that $c \in v$ and $r \in\{1, \ldots, n\} \backslash v$-instead of $X(r, j)$ we write $X\left(r, v_{j}\right)$. Denote by $\mathfrak{R}^{v}$ the set of all such pairs.

The intersection $Y(u)$ of $X(u)$ with the affine patch $\mathbb{A}^{v}$ of $\mathbb{G}_{d, n}$ around $e^{v}$ is of course a closed subvariety in $\mathbb{A}^{v}$. As proved in $[7, \S 5]$, there exist term orders on the monomials in the variables $X(r, c)$ with respect to which the initial ideal of the ideal of functions vanishing on $Y(u)$ is the face ideal of a certain simplicial complex $\mathcal{C}_{u}^{v}$ with vertex set $\mathfrak{R}^{v}$. We want to describe the maximal faces of this complex and thereby the complex itself. 
But before we do that, a digression is necessary. In order that specializations to degenerate situations work smoothly, the correct definition of simplicial complex needs to be adopted. We do not insist, unlike in [12, Chapter II] and like in [11, Definition 1.4], on the axiom that singleton subsets are faces. More precisely, here are our definitions: A simplicial complex is a pair $(V, F)$ of a set $V$ and a set $F$ of subsets of $V$; the elements of $V$ are called vertices and those of $F$ faces; the following axioms hold: (1) the empty subset of $V$ is a face, and (2) a subset of a face is a face. Because of (2) we may replace (1) by (1'): $F$ is non-empty.

Given a simplicial complex $(V, F)$, its (Stanley-Reisner) face ring $R$ is defined as follows: consider the polynomial ring, over some implicit base, in a set of variables indexed by $V$-we abuse notation and let $V$ itself denote the set of variables; the linear span of monomials whose support is not contained in any face forms an ideal - let us call it the face ideal (or should it be the non-face ideal?); the quotient of the polynomial ring by the face ideal is $R$. It is readily seen that the face ideal is the intersection, over all maximal faces, of the ideal generated by the variables in the complement of that face.

The digression being over, we now start on the description of the simplicial complex $\mathcal{C}_{u}^{v}$. Denote by $\mathfrak{N}^{v}$ the subset of $\mathfrak{R}^{v}$ consisting of those pairs $(r, c)$ for which $r>c$. The element $u$ of $I(d, n)$ determines as follows a subset $\mathfrak{S}_{u}^{v}$ of $\mathfrak{N}^{v}$ with the following property: writing $\mathfrak{S}_{u}^{v}=\left\{\left(r_{1}, c_{1}\right), \ldots,\left(r_{k}, c_{k}\right)\right\}$, we have $u=\left(v \backslash\left\{c_{1}, \ldots, c_{k}\right\}\right) \cup\left\{r_{1}, \ldots, r_{k}\right\}$. To define $\mathfrak{S}_{u}^{v}$, proceed by induction on $d$. Let $i, 1 \leq i \leq d$, be the largest such that $v_{i} \leq u_{1}$. Set $v^{\prime}=v \backslash\left\{v_{i}\right\}$ and $u^{\prime}=u \backslash\left\{u_{1}\right\}$. Then $v^{\prime} \leq u^{\prime}$ and $\mathfrak{S}_{u^{\prime}}^{v^{\prime}}$ is defined by induction. Set

$$
\mathfrak{S}_{u}^{v}= \begin{cases}\mathfrak{S}_{u^{\prime}}^{v^{\prime}} \cup\left\{\left(u_{1}, v_{i}\right)\right\} & \text { if } u_{1} \neq v_{i} \\ \mathfrak{S}_{u^{\prime}}^{v^{\prime}} & \text { if } u_{1}=v_{i}\end{cases}
$$

We draw - see Example 1 and Figure 1 below-a grid with the elements of $\mathfrak{N}^{v}$ being the lattice points - in the notation $(r, c)$, the $r$ is suggestive of row index and $c$ of column index. The solid dots in the figure denote the points of $\mathfrak{S}_{u}^{v}$. From each solid dot $\beta$ we draw a vertical line and a horizontal line. Let $\beta$ (start) and $\beta$ (finish) denote respectively the points where the vertical and the horizontal lines meet the boundary. In Figure 1 for example $\beta$ (start $)=(14,11)$ and $\beta$ (finish $)=(16,13)$ for $\beta=(16,11)$.

A lattice path between a pair of such points $\beta$ (start) and $\beta$ (finish) is a sequence $\alpha_{1}, \ldots, \alpha_{q}$ of elements of $\mathfrak{N}^{v}$ with $\alpha_{1}=\beta$ (start), $\alpha_{q}=\beta$ (finish), and for $j, 1 \leq j \leq$ $q-1$, writing $\alpha_{j}=(r, c), \alpha_{j+1}$ is either $\left(r^{\prime}, c\right)$ or $\left(r, c^{\prime}\right)$, where $r^{\prime}$ (respectively $c^{\prime}$ ) is the smallest integer not in $v$ (respectively in $v$ ) and greater than $r$ (respectively $c)$. If $\beta$ (start) $=(r, c)$ and $\beta$ (finish) $=(R, C)$, then $q=(R-r)+(C-c)+1$.

Let us write $\mathfrak{S}_{u}^{v}=\left\{\beta_{1}, \ldots, \beta_{p}\right\}$. Consider the set of all $p$-tuples of paths $\Lambda=\left(\Lambda_{1}, \ldots, \Lambda_{p}\right)$, where $\Lambda_{j}$ is a lattice path between $\beta_{j}$ (start) and $\beta_{j}($ finish $)$, and 


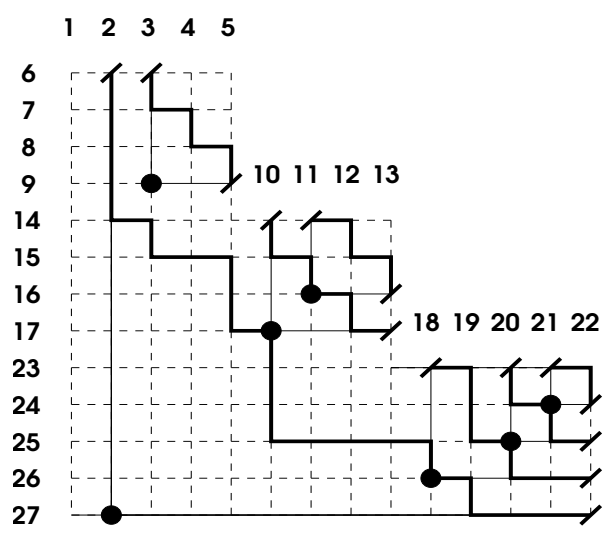

Figure 1. A tuple of non-intersecting lattice paths as in Example 1

no two $\Lambda_{j}$ intersect. A particular such $p$-tuple is shown in Figure 1. Such $p$-tuples form an indexing set for the maximal faces of the simplicial complex $\mathcal{C}_{u}^{v}$ : to the $p$-tuple $\Lambda=\left(\Lambda_{1}, \ldots, \Lambda_{p}\right)$ corresponds the maximal face $\Lambda_{1} \cup \cdots \cup \Lambda_{p} \cup\left(\mathfrak{R}^{v} \backslash \mathfrak{N}^{v}\right)$ : in the degenerate case when $\mathfrak{S}_{u}^{v}$ is empty (which happens only if $u=v$ ), there is a unique maximal face, namely $\mathfrak{R}^{v} \backslash \mathfrak{N}^{v}$.

Example 1. Let $d=14, n=27$,

$$
\begin{aligned}
v & =(1,2,3,4,5,10,11,12,13,18,19,20,21,22), \text { and } \\
u & =(1,4,5,9,12,13,16,17,19,22,24,25,26,27), \text { so that } \\
\mathfrak{S}_{u}^{v} & =\{(9,3),(16,11),(17,10),(24,21),(25,20),(26,18),(27,2)\} .
\end{aligned}
$$

Figure 1 shows a particular tuple of non-intersecting lattice paths.

Consider now the subvariety of $\mathbb{A}^{v}$ defined by the face ideal of the complex $\mathcal{C}_{u}^{v}$. It is a union of coordinate planes. There is one plane for each maximal face and it is defined by the vanishing of the coordinates corresponding to the vertices in the complement of that face. For a maximal face $f$ corresponding to $\left(\Lambda_{1}, \ldots, \Lambda_{p}\right)$, denote by $m_{f}$ the product, over all $(r, c)$ in $\mathfrak{N}^{v} \backslash\left(\Lambda_{1} \cup \cdots \cup \Lambda_{p}\right)$, of $\epsilon(r, c):=\epsilon_{r}-\epsilon_{c}$. It follows from Theorem 3 that the restriction $\left.\left[X_{u}\right]\right|_{v}$ is the sum $\sum m_{f}$ as $f$ varies over all maximal faces. The last assertion holds also in the degenerate case $u=v$ : then $\mathfrak{S}_{u}^{v}$ is empty; $\mathcal{C}_{u}^{v}$ has only one maximal face, namely $\mathfrak{R}^{v} \backslash \mathfrak{N}^{v}$; and $\sum m_{f}$ is the product over $(r, c)$ in $\mathfrak{N}^{v}$ of $\left(\epsilon_{r}-\epsilon_{c}\right)$. In particular,

- If $u=v=(1,2, \ldots, d)$, then $\mathcal{C}_{u}^{v}$ has only the empty face, and $\sum m_{f}$ is the product over $(r, c)$ in $\mathfrak{N}^{v}=\mathfrak{R}^{v}$ of $\left(\epsilon_{r}-\epsilon_{c}\right)$.

- If $u=v=(n-d+1, \ldots, n)$, then the unique maximal face of $\mathcal{C}_{u}^{v}$ is $f=\mathfrak{R}^{v}$ and $m_{f}$, being the product over an empty index set, equals 1. 
Example 2. This example is simple enough so we can easily draw all possible tuples of non-intersecting lattice paths. Let $d=6, n=13$,

$$
\begin{aligned}
& v=(1,2,3,8,9,10), \text { and } u=(4,6,7,10,11,13) . \\
& \text { Then } \mathfrak{S}_{u}^{v}=\{(4,3),(6,2),(7,1),(11,9),(13,8)\} .
\end{aligned}
$$

Figure 2 shows all the 5 -tuples of non-intersecting lattice paths - there are 9 of them. Writing $\epsilon(r, c)$ for $\epsilon_{r}-\epsilon_{c}$,

$$
\begin{aligned}
{\left.\left[X_{u}\right]\right|_{v}=} & \epsilon(11,1) \epsilon(11,2) \epsilon(11,3) \epsilon(12,1) \epsilon(12,2) \epsilon(12,3) \epsilon(13,1) \epsilon(13,2) \epsilon(13,3) . \\
& {[\epsilon(12,9) \epsilon(12,10)+\epsilon(13,8) \epsilon(12,10)+\epsilon(13,8) \epsilon(13,9)] . } \\
& {[\epsilon(5,3)+\epsilon(6,2)+\epsilon(7,1)] . \square }
\end{aligned}
$$
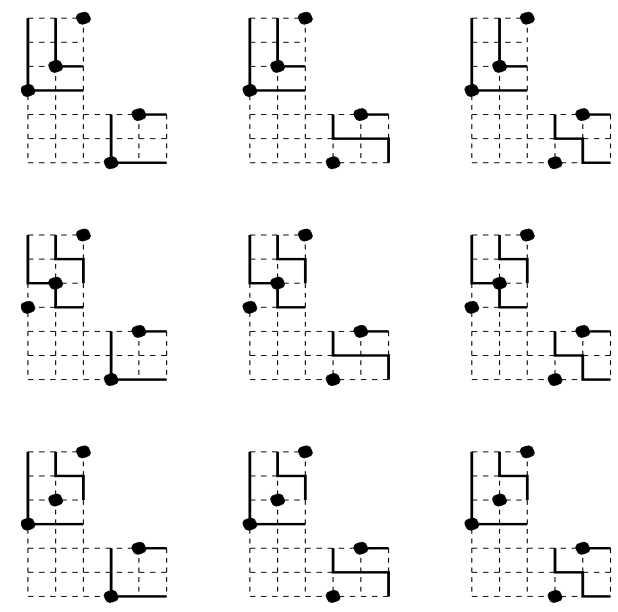

Figure 2. All the tuples of non-intersecting lattice paths as in Example 2

Thus the proof of the restriction formula is reduced to the combinatorial problem of establishing

$$
\sum m_{f}=E(u, v)
$$

where $E(u, v)$ stands for the expression (3). Whether this problem admits of an elegant solution by means of a Lindstrom-Gessel-Viennot type argument we do not know. What follows is an elementary argument based on induction.

Proceed by induction on $d$. The case $d=1$ being easily verified, let $d \geq 2$. The strategy of the proof is this. In the first part, we work with $\sum m_{f}$ and express it in terms of "smaller" $\sum m_{f}$ - those attached to simplicial complexes $\mathcal{C}_{u^{\prime}}^{v^{\prime}}$ for elements $u^{\prime} \geq v^{\prime}$ in $I(d-1, n)$. By the induction hypothesis, equality (7) applies to these smaller $\sum m_{f}$, so that we get an expression for $\sum m_{f}$ in terms of $E\left(u^{\prime}, v^{\prime}\right)$ - the precise expression is in (10) below. In the second part, we will algebraically manipulate the expression $(3)$ for $E(u, v)$ to express it in 


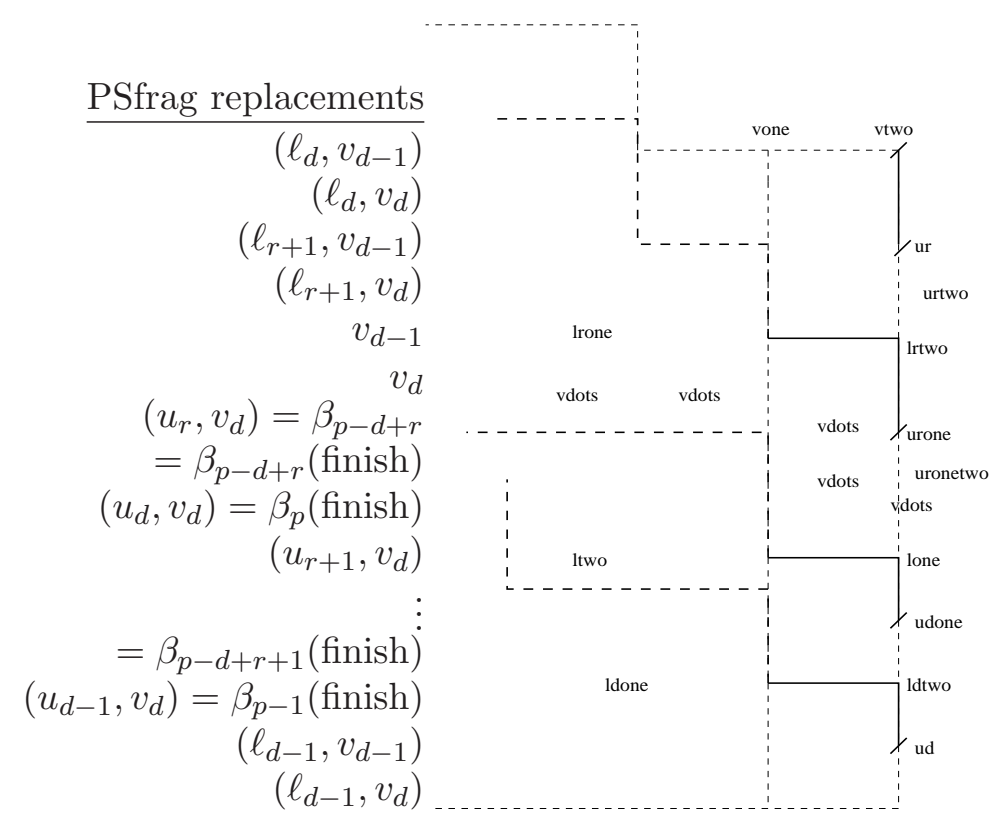

Figure 3. Partitioning the maximal faces of $\mathcal{C}_{u}^{v}$ into $S_{\underline{\ell}}$

terms of $E\left(u^{\prime}, v^{\prime}\right)$. The resulting expression will turn out to be the same as that for $\sum m_{f}$ obtained in the first part. This will finish the proof.

So first consider $\sum m_{f}$. Let $r$ be the integer, $1 \leq r \leq d$, such that $u_{r-1}<v_{d} \leq$ $u_{r}$. Write as before $\mathfrak{S}_{u}^{v}=\left\{\beta_{1}, \ldots, \beta_{p}\right\}$. It is easy to see that

$$
\begin{gathered}
\beta_{p}(\text { finish })=\left(u_{d}, v_{d}\right), \beta_{p-1}(\text { finish })=\left(u_{d-1}, v_{d}\right), \ldots, \\
\qquad \beta_{p-d+r+1}(\text { finish })=\left(u_{r+1}, v_{d}\right) ; \\
\text { furthermore, } \beta_{p-d+r}(\text { finish })=\left(u_{r}, v_{d}\right) \text { unless } u_{r}=v_{d} .
\end{gathered}
$$

Figure 3 depicts the situation.

Partition the set $S$ of the $p$-tuples of paths $\left(\Lambda_{1}, \ldots, \Lambda_{p}\right)$ (those indexing the maximal faces of $\left.\mathcal{C}_{u}^{v}\right)$ into subsets $S_{\underline{\ell}}$ indexed by sequences $\underline{\ell}=\left(\ell_{r+1}, \ldots, \ell_{d}\right)$ of integers such that $u_{r}<\ell_{r+1} \leq u_{r+1}, u_{r+1}<\ell_{r+2} \leq u_{r+2}, \ldots, u_{d-1}<\ell_{d} \leq u_{d}$ : the subset $S_{\ell}$ consists of all those in which the segment joining $\left(\ell_{j}, v_{d-1}\right)$ and $\left(\ell_{j}, v_{d}\right)$ is part of the path $\Lambda_{p-d+j}$ for every $j, r+1 \leq j \leq d$. That the $S_{\underline{\ell}}$ form a partition of $S$ is readily seen. Letting $S_{\underline{\ell}}$ also denote the corresponding partition of the maximal faces of $\mathcal{C}_{u}^{v}$, we obtain

$$
\sum m_{f}=\sum_{\underline{\ell}} \sum_{f \in S_{\underline{\ell}}} m_{f}
$$


For the moment, let us fix a certain $\underline{\ell}$. Set $u^{\prime}:=\left(u_{1}, \ldots, u_{r-1}, l_{r+1}, \ldots, l_{d}\right)$ and $v^{\prime}:=\left(v_{1}, \ldots, v_{d-1}\right)$ (although $u^{\prime}$ depends on $\ell$, we still write only $u^{\prime}$ and not $\left.u^{\prime}(\underline{\ell})\right)$. Then $u^{\prime} \geq v^{\prime}$. We want to use the induction hypothesis to express $\sum_{f \in S_{\ell}} m_{f}$ in terms of $E\left(u^{\prime}, v^{\prime}\right)$. Towards this, we make two observations. First, the factor

$$
\mu_{n}^{u_{d}+1}\left(v_{d}\right) \cdot \prod_{r \leq i<d} \mu_{\ell_{i+1}-1}^{u_{i}+1}\left(v_{d}\right)
$$

is common to all the terms in $\sum_{f \in S_{\ell}} m_{f}$. Second, the integer $v_{d}$ does not occur as a row or column index if we restrict attention to the first $d-1$ columns of Figure 3 (which tells us that $E\left(u^{\prime}, v^{\prime}\right)$ needs to be adjusted to take care of this).

Consider $E\left(u^{\prime}, v^{\prime}\right)$ - this is the expression (3) with $u$ and $v$ replaced respectively by $u^{\prime}$ and $v^{\prime}$. In the matrix whose determinant is the numerator of $E\left(u^{\prime}, v^{\prime}\right)$, the entry in position $(i, j)$ where $i \leq r-1$ has $\epsilon\left(v_{d}, v_{j}\right)$ occurring as a factor-this factor does not occur if $i \geq r$. Denote by $E\left(u^{\prime}, v^{\prime} ; v_{d}\right)$ the modified expression where the factors $\epsilon\left(v_{d}, v_{j}\right)$ are taken out-more precisely, $E\left(u^{\prime}, v^{\prime} ; v_{d}\right):=$

$$
\frac{\left|\begin{array}{ccccc}
\frac{\mu_{n}^{u_{1}+1}\left(v_{1}\right)}{\epsilon\left(v_{d}, v_{1}\right)} & \ldots & \frac{\mu_{n}^{u_{1}+1}\left(v_{j}\right)}{\epsilon\left(v_{d}, v_{j}\right)} & \ldots & \frac{\mu_{n}^{u_{1}+1}\left(v_{d-1}\right)}{\epsilon\left(v_{d}, v_{d-1}\right)} \\
\vdots & \vdots & \vdots & \vdots & \vdots \\
\frac{\mu_{n}^{u_{r-1}+1}\left(v_{1}\right)}{\epsilon\left(v_{d}, v_{1}\right)} & \ldots & \frac{\mu_{n}^{u_{r-1}+1}\left(v_{j}\right)}{\epsilon\left(v_{d}, v_{j}\right)} & \ldots & \frac{\mu_{n}^{u_{r-1}+1}\left(v_{d-1}\right)}{\epsilon\left(v_{d}, v_{d-1}\right)} \\
\mu_{n}^{l_{r+1}+1}\left(v_{1}\right) & \ldots & \mu_{n}^{l_{r+1}+1}\left(v_{j}\right) & \ldots & \mu_{n}^{l_{r+1}+1}\left(v_{d-1}\right) \\
\vdots & \vdots & \vdots & \vdots & \vdots \\
\mu_{n}^{l_{d}+1}\left(v_{1}\right) & \ldots & \mu_{n}^{l_{d}+1}\left(v_{j}\right) & \ldots & \mu_{n}^{l_{d}+1}\left(v_{d-1}\right)
\end{array}\right|}{\epsilon\left(v_{2}, v_{1}\right) \cdot\left(\epsilon\left(v_{3}, v_{1}\right) \epsilon\left(v_{3}, v_{2}\right)\right) \cdot \ldots \cdot\left(\epsilon\left(v_{d-1}, v_{1}\right) \ldots \epsilon\left(v_{d-1}, v_{d-2}\right)\right)}
$$

Looking at Figure 3 and using the induction hypothesis, we get

$$
\sum_{f \in S_{\underline{\ell}}} m_{f}=E\left(u^{\prime}, v^{\prime} ; v_{d}\right) \cdot \mu_{n}^{u_{d}+1}\left(v_{d}\right) \cdot \prod_{r \leq i<d} \mu_{\ell_{i+1}-1}^{u_{i}+1}\left(v_{d}\right) .
$$

We are done with the first half of the proof. Namely, we are finished with the "combinatorial side" $\sum m_{f}$ of Equation (7). Next we turn to the "algebraic side" $E(u, v)$ and show that it too is a sum of terms indexed by the sequences $\underline{\ell}$. We show that the term corresponding to a sequence $\underline{\ell}$ equals the right hand side of (10). This clearly suffices to complete the proof.

By definition, $E(u, v)$ is the expression (3). The entries in the last column of the numerator vanish in rows $i$ for $1 \leq i \leq r-1$ because $u_{i}+1 \leq u_{r-1}+1 \leq v_{d}$ and $0=\epsilon_{v_{d}}-\epsilon_{v_{d}}$ occurs as a factor in $\mu_{n}^{u_{i}+1}\left(v_{d}\right)$. We would like to kill also the 
entries in rows $r$ through $d-1$. To this end, subtract from row $i, r \leq i \leq d-1$, the multiple of row $d$ by $\mu_{u_{d}}^{u_{i}+1}\left(v_{d}\right)$. The entry in position $(i, j)$ then becomes

$$
\mu_{n}^{u_{i}+1}\left(v_{j}\right)-\mu_{u_{d}}^{u_{i}+1}\left(v_{d}\right) \mu_{n}^{u_{d}+1}\left(v_{j}\right)=\mu_{n}^{u_{d}+1}\left(v_{j}\right)\left(\mu_{u_{d}}^{u_{i}+1}\left(v_{j}\right)-\mu_{u_{d}}^{u_{i}+1}\left(v_{d}\right)\right) .
$$

In particular, all the entries in the last column except the one on row $d$ are 0.

The factor $\mu_{n}^{u_{d}+1}\left(v_{j}\right)$ being now common to all the entries in column $j$, let us take these factors out of every column. The resulting entries in the last column are all zero except the one on row $d$ which is 1 . The numerator therefore reduces to the determinant of the submatrix of the first $d-1$ rows and columns.

Let us take the factors $\left(\epsilon_{v_{d}}-\epsilon_{v_{1}}\right), \ldots,\left(\epsilon_{v_{d}}-\epsilon_{v_{d-1}}\right)$ out of the denominator and distribute them thus: divide by $\left(\epsilon_{v_{d}}-\epsilon_{v_{j}}\right)$ every entry in column $j$ of the determinant in the numerator. After these manipulations, expression (3) looks like this:

$$
\prod_{j=1}^{d} \mu_{n}^{u_{d}+1}\left(v_{j}\right) \frac{\operatorname{det}(A)}{\epsilon\left(v_{2}, v_{1}\right)\left(\epsilon\left(v_{3}, v_{1}\right) \epsilon\left(v_{3}, v_{2}\right)\right) \cdots\left(\epsilon\left(v_{d-1}, v_{1}\right) \ldots \epsilon\left(v_{d-1}, v_{d-2}\right)\right)}
$$

where $A:=\left(A_{i j}\right)$ is the $d-1 \times d-1$ matrix whose entry at position $(i, j)$ is

$$
A_{i j}=\frac{\mu_{u_{d}}^{u_{i}+1}\left(v_{j}\right)-\mu_{u_{d}}^{u_{i}+1}\left(v_{d}\right)}{\epsilon_{v_{d}}-\epsilon_{v_{j}}} .
$$

Now apply the following row operations to the matrix $A$ : subtract row $r+1$ from row $r, \ldots$, row $d-1$ from row $d-2$. To get a handle on the resulting matrix - let us call it $B$-we use the following equation which is proved readily by induction: for positive integers $a \leq e \leq b, c$, and $f$, we have

$$
\begin{aligned}
& \frac{\mu_{b}^{a}(c)-\mu_{b}^{a}(f)}{\epsilon_{f}-\epsilon_{c}}= \mu_{b}^{a+1}(c)+\mu_{b}^{a+2}(c) \mu_{a}^{a}(f)+\cdots+\mu_{b}^{e}(c) \mu_{e-2}^{a}(f)+ \\
& \frac{\mu_{b}^{e}(c)-\mu_{b}^{e}(f)}{\epsilon_{f}-\epsilon_{c}} \\
&=\left(\sum_{a-1<\ell \leq e-1} \mu_{b}^{\ell+1}(c) \cdot \mu_{\ell-1}^{a}(f)\right)+\frac{\mu_{b}^{e}(c)-\mu_{b}^{e}(f)}{\epsilon_{f}-\epsilon_{c}}
\end{aligned}
$$

Applying this to (12) for $i$ such that $r \leq i \leq d-1$ and $e=u_{i+1}+1$, we get

$$
\begin{gathered}
\frac{\mu_{u_{d}}^{u_{i}+1}\left(v_{j}\right)-\mu_{u_{d}}^{u_{i}+1}\left(v_{d}\right)}{\epsilon_{v_{d}}-\epsilon_{v_{j}}}=\left(\sum_{u_{i}<\ell_{i+1} \leq u_{i+1}} \mu_{u_{d}}^{\ell_{i+1}+1}\left(v_{j}\right) \cdot \mu_{\ell_{i+1}-1}^{u_{i}+1}\left(v_{d}\right)\right)+ \\
\frac{\mu_{u_{d}}^{u_{i+1}+1}\left(v_{j}\right)-\mu_{u_{d}}^{u_{i+1}+1}\left(v_{d}\right)}{\epsilon_{v_{d}}-\epsilon_{v_{j}}}
\end{gathered}
$$

where we have written $\ell_{i+1}$ rather than just $\ell$ for the running index in the sum. Note that the second term on the right is precisely the entry at position $(i+1, j)$ 
of $A$ for $r \leq i \leq d-2$ and vanishes for $i=d-1$. Thus the entry at position $(i, j)$ of the matrix $B$ looks like this:

$$
B_{i j}= \begin{cases}\frac{\mu_{u_{d}}^{u_{i}+1}\left(v_{j}\right)}{\epsilon\left(v_{d}, v_{j}\right)} & \text { if } i<r \\ \sum_{u_{i}<\ell_{i+1} \leq u_{i+1}} \mu_{u_{d}}^{l_{i+1}+1}\left(v_{j}\right) \cdot \mu_{\ell_{i+1}-1}^{u_{i}+1}\left(v_{d}\right) & \text { if } i \geq r .\end{cases}
$$

By the multilinearity of the determinant, we see that $\operatorname{det}(B)$ (which equals $\operatorname{det}(A)$, since $B$ was obtained from $A$ by elementary row operations) equals the sum over $\underline{\ell}=\left(\ell_{r+1}, \ldots, \ell_{d}\right)$ of

$$
\prod_{r \leq i<d} \mu_{\ell_{i+1}-1}^{u_{i}+1}\left(v_{d}\right) \cdot\left|\begin{array}{ccccc}
\frac{\mu_{u_{d}}^{u_{1}+1}\left(v_{1}\right)}{\epsilon\left(v_{d}, v_{1}\right)} & \ldots & \frac{\mu_{u_{d}}^{u_{1}+1}\left(v_{j}\right)}{\epsilon\left(v_{d}, v_{j}\right)} & \ldots & \frac{\mu_{u_{d}}^{u_{1}+1}\left(v_{d-1}\right)}{\epsilon\left(v_{d}, v_{d-1}\right)} \\
\vdots & \vdots & \vdots & \vdots & \vdots \\
\frac{\mu_{u_{d}}^{u_{r-1}+1}\left(v_{1}\right)}{\epsilon\left(v_{d}, v_{1}\right)} & \ldots & \frac{\mu_{u_{d}}^{u_{r-1}+1}\left(v_{j}\right)}{\epsilon\left(v_{d}, v_{j}\right)} & \ldots & \frac{\mu_{u_{d}}^{u_{r-1}+1}\left(v_{d-1}\right)}{\epsilon\left(v_{d}, v_{d-1}\right)} \\
\mu_{u_{d}}^{l_{r+1}+1}\left(v_{1}\right) & \ldots & \mu_{u_{d}}^{l_{r+1}+1}\left(v_{j}\right) & \ldots & \mu_{u_{d}}^{l_{r+1}+1}\left(v_{d-1}\right) \\
\vdots & \vdots & \vdots & \vdots & \vdots \\
\mu_{u_{d}}^{l_{d}+1}\left(v_{1}\right) & \ldots & \mu_{u_{d}}^{l_{d}+1}\left(v_{j}\right) & \ldots & \mu_{u_{d}}^{l_{d}+1}\left(v_{d-1}\right)
\end{array}\right| .
$$

Substitute this into equation (11). Multiplying the factor $\mu_{n}^{u_{d}+1}\left(v_{j}\right)$, for $1 \leq j \leq$ $d-1$, in equation (11) into all the entries in column $j$ of the determinant in (13) yields the determinant in the numerator of $E\left(u^{\prime}, v^{\prime} ; v_{d}\right)$ in $(9)$. It should now be clear that $E(u, v)$ is the sum over $\underline{\ell}$ of the right side of (10), and the proof of the restriction formula (Theorem 2 ) is finally over.

\section{Gröbner Degeneration COMPutes REStriction}

The goal of this section is to state and prove Theorem 3 below, which was used in the proof in $\S 5$ of the restriction formula (Theorem 2). As pointed out to us by the referee, Theorem 3 is well known and can be deduced from results in the literature.

The assumptions and notations of the previous sections are annulled now. Fix a torus $T:=\left(\mathbb{C}^{*}\right)^{m}$. Let $Z$ be a non-singular complex projective variety of dimension $d$ on which there is an algebraic action of $T$ with finitely many fixed points. Then, by Bialynicki-Birula [1], $Z$ admits an equivariant algebraic cell decomposition, and around each $T$-fixed point there is a $T$-stable open subset $U$ of $Z$ that is isomorphic to a $T$-module (the fixed point of course corresponds to 0 in the module). 
Let $Y$ be a $T$-stable irreducible subvariety of $Z, y$ a $T$-fixed point on $Y$, and $U \simeq \mathbb{C}^{d}$ a $T$-stable open subset of $Z$ containing $y$ as above. Let $X_{1}, \ldots, X_{d}$ be coordinates on $U$ that are eigenvectors for $T$ - since $y$ is an isolated fixed point, no coordinate has trivial character. Choose some term order on the set of monomials in the coordinates, and suppose that $J$ is the initial ideal, with respect to this order, of the ideal of functions on $U$ vanishing on $Y \cap U$.

Since $J$ is a monomial ideal, it has a primary decomposition consisting of monomial ideals. Let $\cap_{i=1}^{p} J_{i}$ be the intersection of the minimal primary components (we are throwing away the embedded components). The radical $\mathfrak{p}_{i}$ of $J_{i}$ is of the form $\left(X_{1}^{a_{1 i}}, \ldots, X_{d}^{a_{d i}}\right)$ where each $a_{j i}$ is either 0 or 1 (exactly $\operatorname{dim} Y$ of the $a_{j i}$ equal 0 for each $i$ ). The scheme $M$ defined by $\cap_{i=1}^{p} J_{i}$ is the union $\cup_{i=1}^{p} M_{i}$ of the schemes $M_{i}$ defined by $J_{i}$. Let $\ell_{i}$ be the length of $R_{\mathfrak{p}_{i}} / J R_{\mathfrak{p}_{i}}=R_{\mathfrak{p}_{i}} / J_{i} R_{\mathfrak{p}_{i}}$ where $R:=\mathbb{C}\left[X_{1}, \ldots, X_{d}\right]$.

Theorem 3. With hypothesis and notation as above, the restriction to the fixed point $y$ of the equivariant cohomology class $[Y]$ of the subvariety $Y$ in the equivariant integral cohomology ring of $Z$ is given by

$$
\left.[Y]\right|_{y}=\sum_{i=1}^{p} \ell_{i} \prod_{j=1}^{d} \chi_{j}^{a_{j i}}
$$

where $-\chi_{1}, \ldots,-\chi_{d}$ are respectively the characters of $X_{1}, \ldots, X_{d}$.

Proof : We thank the referee for indicating how the theorem can be deduced as follows from known results. The restriction of the equivariant Chow class of $Y$ to the fixed point $y$ factors through the restriction to the open set $U$. The fact that the class of $Y \cap U$ and of $M$ in the equivariant Chow ring of $U$ are the same and equal to the right hand side of (14) can be found in any number of references under the heading of "equivariant Chow", or "multidegree", or "equivariant Hilbert polynomials", or "equivariant multiplicity." See, for example, [11, Notes to Chapter 8]; the fact that the "multidegrees" in the above reference are equivariant cohomology classes is asserted in Proposition 1.19 of [4], where multidegrees are identified as being equivariant Chow classes.

\section{TOWARDS AN EQUIVARIANT PIERI FORMUla}

Recall, from [3, Eq. (9), p.146] for example, that the classical Pieri formula gives a beautiful expression, as an integral linear combination of general Schubert classes, for the product of a special Schubert class with a general Schubert class in the ordinary cohomology ring of the Grassmannian. It seems like there ought to be a similarly beautiful closed-form equivariant version that specializes to the ordinary one. Unfortunately, this we do not yet have. 
All we want to do in this section is record an observation (see Proposition below) that is a formal consequence of the following basic and well-known facts: the injection of Equation (1); the restriction to a $T$-fixed point of an (equivariant) Schubert class vanishes if the fixed point is not contained in the Schubert variety; and the degree of a Schubert class equals the codimension of the Schubert variety. Being a formal consequence, the observation holds for any generalized flag variety, not just the Grassmannian. The point to note is that the right hand side of (15) is in terms of restrictions, which, thanks to Theorem 2, we know how to compute in the case of Grassmannians.

Let $G$ be a complex semisimple algebraic group and $Q$ a parabolic subgroup. Let $T$ be a maximal torus and $B$ a Borel subgroup of $G$ such that $T \subseteq B \subseteq Q$. Let $W$ denote the Weyl group of $G$ with respect to $T$, and $W_{Q}$ the Weyl group of (the Levi part of) $Q$ with respect to $T$. The Schubert varieties in $G / Q$ are by definition the $B$-orbit closures for the action of $B$ on $G / Q$ by left multiplication. They are naturally indexed by $W / W_{Q}$. We use $u, v, w, \ldots$ to denote elements of $W / W_{Q} ; X(u), X(v), X(w), \ldots$ denote the corresponding Schubert varieties; $[X(u)],[X(v)],[X(w)], \ldots$ denote the corresponding equivariant Schubert classes.

The partial order on Schubert varieties by inclusion induces a partial order, denoted $\leq$, on $W / W_{Q}$. Let $c_{u v}^{w}$ be the structure constants of the multiplication of the equivariant integral cohomology ring of $G / Q$ with the respect to the basis of Schubert classes:

$$
[X(u)] \cdot[X(v)]=\sum_{w} c_{u v}^{w}[X(w)]
$$

The proof of the following proposition is a straightforward induction argument and so we omit it.

Proposition 4. (1) $c_{u v}^{w}=0$ unless $w \leq u, w \leq v$, and the codimensions are such that $\operatorname{codim} X(u)+\operatorname{codim} X(v) \geq \operatorname{codim} X(w)$.

(2) If $w \leq u$ and $w \leq v$, then

(15) $c_{u v}^{w}=\sum_{w=y_{0}<\ldots<y_{k}}(-1)^{k} \cdot \frac{\left.\left.[X(u)]\right|_{y_{k}}[X(v)]\right|_{y_{k}}}{\left.\left[X\left(y_{k}\right)\right]\right|_{y_{k}}} \cdot \frac{\left.\left[X\left(y_{k}\right)\right]\right|_{y_{k-1}}}{\left.\left[X\left(y_{k-1}\right)\right]\right|_{y_{k-1}}} \cdots \cdot \frac{\left.\left[X\left(y_{1}\right)\right]\right|_{y_{0}}}{\left.\left[X\left(y_{0}\right)\right]\right|_{y_{0}}}$ where the sum is over all chains $w=y_{0}<\ldots<y_{k}$ with $y_{k} \leq u$ and $y_{k} \leq v ;\left.[X(u)]\right|_{y_{k}}$ denotes the restriction of the Schubert class $[X(u)]$ to the $T$-fixed point indexed by $y_{k}$.

\section{REFERENCES}

1. A. Bialynicki-Birula, Some theorems on actions of algebraic groups, Ann. Math. 98 (1973), 480-497.

2. W. Fulton, Intersection Theory, Ergebnisse der Mathematik und ihrer Grenzgebiete, 3 Folge-Band 2, A Series of Modern Surveys in Mathematics, Springer-Verlag, 1984. 
3. W. Fulton, Young Tableaux, London Mathematical Society Student Texts, Cambridge University Press, 1997.

4. A. Knutson, E. Miller, M. Shimozono, Four positive formulae for type A quiver polynomials, arXiv:math.AG/0308142.

5. A. Knutson, T. Tao, C. Woodward, The honeycomb model of $\mathrm{GL}_{n}(\mathbb{C})$ tensor products. II. Puzzles determine facets of the Littlewood-Richardson cone, J. Amer. Math. Soc. 17 (2004), no. 1, 19-48 (electronic).

6. A. Knutson and T. Tao, Puzzles and (equivariant) cohomology of Grassmannians, Duke Math Journal 119 (2003), no. 2, 221-260.

7. V. Kodiyalam and K. N. Raghavan, Hilbert functions of points on Schubert varieties in Grassmannians, J. Algebra, 270 (2003), 28-54.

8. V. Kreiman, Monomial Bases and Applications for Richardson and Schubert Varieties in Ordinary and Affine Grassmannians, Thesis, Northeastern University, 2003.

9. V. Kreiman and V. Lakshmibai, Multiplicities of Singular Points of Schubert Varieties of Grassmannians, Algebra, Arithmetic and Geometry with Applications (West Lafayette, IN, 2000), pp. 553-563, Springer, Berlin, 2004.

10. V. Kreiman and V. Lakshmibai, Richardson Varieties in the Grassmannians, Contributions to Automorphic Forms, Geometry and Number Theory (Baltimore, MD, 2002), pp. 573-597, Johns Hopkins Univ. Press, Baltimore, MD, 2004.

11. E. Miller and B. Sturmfels, Combinatorial Commutative Algebra, Springer GTM Vol. 227, 2005.

12. R. Stanley, Combinatorics and Commutative Algebra, Progress in Mathematics Vol. 41, Birkhäuser, 1983.

V. Lakshmibai

Department of Mathematics

Northeastern University

Boston, MA02115

E-mail: lakshmibai@neu.edu

K. N. Raghavan

Institute of Mathematical Sciences

C.I.T. Campus Chennai 600113 INDIA

E-mail: knr@imsc.res.in

P. Sankaran

Institute of Mathematical Sciences

C.I.T. Campus, Chennai 600113 INDIA

E-mail: sankaran@imsc.res.in 